\title{
Efficiency and Generalized Convex Duality for Nondifferentiable Multiobjective Programs
}

\author{
Kwan Deok Bae, Young Min Kang, and Do Sang Kim \\ Division of Mathematical Sciences, Pukyong National University, Busan 608-737, South Korea \\ Correspondence should be addressed to Do Sang Kim, dskim@pknu.ac.kr
}

Received 29 October 2009; Accepted 15 February 2010

Academic Editor: Jong Kyu Kim

Copyright (C) 2010 Kwan Deok Bae et al. This is an open access article distributed under the Creative Commons Attribution License, which permits unrestricted use, distribution, and reproduction in any medium, provided the original work is properly cited.

We introduce nondifferentiable multiobjective programming problems involving the support function of a compact convex set and linear functions. The concept of (properly) efficient solutions are presented. We formulate Mond-Weir-type and Wolfe-type dual problems and establish weak and strong duality theorems for efficient solutions by using suitable generalized convexity conditions. Some special cases of our duality results are given.

\section{Introduction and Preliminaries}

The concept of efficiency has long played an important role in economics, game theory, statistical decision theory, and in all optimal decision problems with noncomparable criteria. In 1968, Geoffrion [1] proposed a slightly restricted definition of efficiency that eliminates efficient points of a certain anomalous type and lended itself to more satisfactory characterization. He called this new definition proper efficiency. Weir [2] has used proper efficiency to establish some duality results between primal problem and Wolfe type dual problem. He extended the duality results of Wolfe [3] for scalar convex programming problems and some of the more duality results for scalar nonconvex programming problems to vector valued programs.

In 1982, five characterizations of strongly convex sets were introduced by Vial [4]. Based on this, Vial [5] studied a class of functions depending on the sign of the constant $\rho$. Characteristic properties of this class of sets and related it to strong and weakly convex sets are provided.

Also, Egudo [6] and Weir [2] have used proper efficiency to obtain duality relations between primal problem and Mond-Weir type dual problem. Further, Egudo [7] used the concept of efficiency to formulate duality for multiobjective non-linear programs under generalized $\rho$-convexity assumptions. 
Duality theorems for nondifferentiable programming problem with a square root term were obtained by Lal et al. [8]. In 1996, Mond and Schechter [9] studied duality and optimality for nondifferentiable multiobjective programming problems in which each component of the objective function contains the support functions of a compact convex sets. And Kuk et al. [10] defined the concept of $(V, \rho)$-invexity for vector-valued functions, which is a generalization of the concept of $V$-invexity concept.

Recently, Yang et al. [11] introduced a class of nondifferentiable multiobjective programming problems involving the support functions of compact convex sets. They established only weak duality theorems for efficient solutions. Subsequently, Kim and Bae [12] formulated nondifferentiable multiobjective programs involving the support functions of a compact convex sets and linear functions.

In this paper, we introduce generalized convex duality for nondifferentiable multiobjective program for efficient solutions. In Section 2 and Section 3, we formulate MondWeir type dual and Wolfe type dual problems and establish weak and strong duality under $\rho$-convexity assumptions. In addition, we obtain some special cases of our duality results in Section 4 . Our duality results extend and improve well known duality results.

We consider the following multiobjective programming problem:

$$
\begin{array}{ll}
\operatorname{minimize} & \left(f_{1}(x)+s\left(x \mid C_{1}\right), \ldots, f_{p}(x)+s\left(x \mid C_{p}\right)\right) \\
\text { subject to } & g_{j}(x) \leqq 0, \quad j=1, \ldots, m, \\
& h_{l}(x)=0, \quad l=1, \ldots, q .
\end{array}
$$

The functions $f_{i}: \mathbb{R}^{n} \rightarrow \mathbb{R}, i=1, \ldots, p, g_{j}: \mathbb{R}^{n} \rightarrow \mathbb{R}, j=1, \ldots, m$ and $h_{l}: \mathbb{R}^{n} \rightarrow \mathbb{R}, l=$ $1, \ldots, q$ are assumed to be differentiable. And $C_{i}$, for each $i \in P=\{1,2, \ldots, p\}$, is a compact convex set of $\mathbb{R}^{n}$.

Definition 1.1. A feasible solution $x^{0}$ for (VOPE) is efficient for (VOPE) if and only if there is no other feasible $x$ for (VOPE) such that

$$
\begin{gathered}
f_{i}(x)+s\left(x \mid C_{i}\right)<f_{i}\left(x^{0}\right)+s\left(x^{0} \mid C_{i}\right) \quad \text { for some } i \in P, \\
f_{i_{0}}(x)+s\left(x \mid C_{i_{0}}\right) \leqq f_{i_{0}}\left(x^{0}\right)+s\left(x^{0} \mid C_{i_{0}}\right) \quad \forall i_{0} \in P .
\end{gathered}
$$

Definition 1.2. Let $C$ be a compact convex set in $\operatorname{IR}^{n}$. The support function $s(x \mid C)$ is defined by

$$
s(x \mid C):=\max \left\{x^{T} y: y \in C\right\}
$$

The support function $s(x \mid C)$, being convex and everywhere finite, has a subdifferential, that is, there exists $z$ such that

$$
s(y \mid C) \geq s(x \mid C)+z^{T}(y-x) \quad \forall y \in C .
$$


Equivalently,

$$
z^{T} x=s(x \mid C)
$$

The subdifferential of $s(x \mid C)$ is given by

$$
\partial s(x \mid C):=\left\{z \in C: z^{T} x=s(x \mid C)\right\}
$$

The following definition of $\rho$-convex function will be used to prove weak duality theorems in Section 2 and Section 3.

Definition 1.3 (see $[4,5]$ ). A function $f_{i}: \mathbb{R}^{n} \rightarrow \mathbb{R}$ is said to be $\rho_{i}$-convex if there exists a real number $\rho_{i}$ such that for each $x, u \in \mathbb{R}^{n}$ and $0 \leqq \lambda_{i} \leqq 1$,

$$
f_{i}\left(\lambda_{i} x+\left(1-\lambda_{i}\right) u\right) \leqq \lambda_{i} f_{i}(x)+\left(1-\lambda_{i}\right) f_{i}(u)-\rho_{i} \lambda_{i}\left(1-\lambda_{i}\right)\|x-u\|^{2}
$$

For a differentiable function $f_{i}: \mathbb{R}^{n} \rightarrow \mathbb{R}, f_{i}$ is $\rho_{i}$-convex if and only if for all $x, u \in \mathbb{R}^{n}$,

$$
f_{i}(x)-f_{i}(u) \geqq(x-u)^{T} \nabla f_{i}(u)+\rho_{i}\|x-u\|^{2} .
$$

If $\rho_{i}$ is positive then $f_{i}$ is said to be strongly convex [4] and if $\rho_{i}$ is negative then $f_{i}$ is said to be weakly convex [5].

In this paper, the proofs of strong duality theorems will invoke the following.

Lemma 1.4. (Chankong and Haimes [13, Theorem 4.1]) $x^{0}$ is an efficient solution for (VOPE) if and only if $x^{0}$ solves the following:

$$
\begin{aligned}
\left(P_{k}\left(\epsilon^{0}\right)\right) \quad \text { minimize } & f_{k}(x)+s\left(x \mid C_{k}\right) \\
\text { subject to } & f_{i}(x)+s\left(x \mid C_{i}\right) \\
& \leqq f_{i}\left(x^{0}\right)+s\left(x^{0} \mid C_{i}\right), \quad \forall i \neq k, \\
& g_{j}(x) \leqq 0, \quad j=1, \ldots, m, \\
& h_{l}(x)=0, \quad l=1, \ldots, q
\end{aligned}
$$

for each $k=1, \ldots, p$. 


\section{Mond-Weir-Type Duality}

We introduce a Mond-Weir type dual programming problem and establish weak and strong duality theorems.

$$
\begin{array}{ll}
\text { Maximize } & f(u)+u^{T} w=\left(f_{1}(u)+u^{T} w_{1}, \ldots, f_{p}(u)+u^{T} w_{p}\right) \\
\text { subject to } & \\
& \sum_{i=1}^{p} \tau_{i}\left(\nabla f_{i}(u)+w_{i}\right)+\nabla y^{T} g(u)+\nabla z^{T} h(u)=0, \\
& y^{T} g(u)+z^{T} h(u) \geqq 0, \\
& y_{j} \geqq 0, \quad j=1, \ldots, m, w_{i} \in C_{i}, i=1, \ldots, p, \\
& \tau_{i} \geqq 0, \quad i=1, \ldots, p, \quad \sum_{i=1}^{p} \tau_{i}=1 .
\end{array}
$$

(MVODE)

Theorem 2.1 (Weak Duality). Assume that for all feasible $x$ for (VOPE) and all feasible $(u, \tau, w, y, z)$ for (MVODE), $f_{i}(\cdot)+(\cdot)^{T} w_{i}, i=1, \ldots, p$, are $\rho_{i}$-convex $, g_{j}(\cdot), j=1, \ldots, m$, are $\sigma_{j}$-convex and $h_{l}(\cdot), l=1, \ldots, q$, are affine. If also any of the following conditions holds

(a) $\tau_{i}>0$, forall $i \in P$ and $\sum_{i=1}^{p} \tau_{i} \rho_{i}+\sum_{j=1}^{m} y_{j} \sigma_{j} \geqq 0$;

(b) $\sum_{i=1}^{p} \tau_{i} \rho_{i}+\sum_{j=1}^{m} y_{j} \sigma_{j}>0$,

then the following cannot hold:

$$
\begin{gathered}
f_{i}(x)+s\left(x \mid C_{i}\right)<f_{i}(u)+u^{T} w_{i}, \quad \text { for some } i \in P \\
f_{i_{0}}(x)+s\left(x \mid C_{i_{0}}\right) \leqq f_{i_{0}}(u)+u^{T} w_{i_{0}}, \quad \forall i_{0} \in P .
\end{gathered}
$$

Proof. Suppose contrary to the result that (2.3) hold; then for $\tau_{i}>0$ for each $i=1, \ldots, p,(2.3)$ imply

$$
\sum_{i=1}^{p} \tau_{i}\left(f_{i}(x)+s\left(x \mid C_{i}\right)\right)<\sum_{i=1}^{p} \tau_{i}\left(f_{i}(u)+u^{T} w_{i}\right) \quad \text { for some } i \in P
$$

and for $\tau_{i} \geqq 0, i=1, \ldots, p,(2.3)$ imply

$$
\sum_{i=1}^{p} \tau_{i}\left(f_{i_{0}}(x)+s\left(x \mid C_{i_{0}}\right)\right) \leqq \sum_{i=1}^{p} \tau_{i}\left(f_{i_{0}}(u)+u^{T} w_{i_{0}}\right) \quad \forall i_{0} \in P .
$$

Since $x^{T} w_{i} \leqq s\left(x \mid C_{i}\right), \quad i=1, \ldots, p$, then (2.4) and (2.5) imply

$$
\begin{gathered}
\sum_{i=1}^{p} \tau_{i}\left(f_{i}(x)+x^{T} w_{i}\right)<\sum_{i=1}^{p} \tau_{i}\left(f_{i}(u)+u^{T} w_{i}\right), \\
\sum_{i=1}^{p} \tau_{i}\left(f_{i_{0}}(x)+x^{T} w_{i_{0}}\right) \leqq \sum_{i=1}^{p} \tau_{i}\left(f_{i_{0}}(u)+u^{T} w_{i_{0}}\right) .
\end{gathered}
$$


From $\rho_{i}$-convexity of $f_{i}(\cdot)+(\cdot)^{T} w_{i}, i=1, \ldots, p$, we have

$$
\begin{gathered}
(x-u)^{T}\left(\sum_{i=1}^{p} \tau_{i}\left(\nabla f_{i}(u)+w_{i}\right)\right)+\left(\sum_{i=1}^{p} \tau_{i} \rho_{i}\right)\|x-u\|^{2}<0 \quad \text { for some } i \in P, \\
(x-u)^{T}\left(\sum_{i=1}^{p} \tau_{i}\left(\nabla f_{i_{0}}(u)+w_{i_{0}}\right)\right)+\left(\sum_{i=1}^{p} \tau_{i} \rho_{i}\right)\|x-u\|^{2} \leqq 0 \quad \forall i_{0} \in P
\end{gathered}
$$

respectively. Also, since $(u, \tau, w, y, z)$ is feasible for (MVODE) and $x$ is feasible for (VOPE), we have

$$
\left(\sum_{j=1}^{m} y_{j} g_{j}(x)+\sum_{l=1}^{q} z_{l} h_{l}(x)\right)-\left(\sum_{j=1}^{m} y_{j} g_{j}(u)+\sum_{l=1}^{q} z_{l} h_{l}(u)\right) \leqq 0 .
$$

Since $g_{j}(\cdot), j=1, \ldots, m$ are $\sigma_{j}$-convex and $h_{l}(\cdot), l=1, \ldots, q$ are affine, then (2.9) imply

$$
(x-u)^{T}\left(\sum_{j=1}^{m} y_{j} \nabla g_{j}(u)+\sum_{l=1}^{q} z_{l} \nabla h_{l}(u)\right)+\left(\sum_{j=1}^{m} y_{j} \sigma_{j}\right)\|x-u\|^{2} \leqq 0 .
$$

Adding (2.7), (2.10) and then applying hypothesis (a), we get

$$
(x-u)^{T}\left(\sum_{i=1}^{p} \tau_{i}\left(\nabla f_{i}(u)+w_{i}\right)+\sum_{j=1}^{m} y_{j} \nabla g_{j}(u)+\sum_{l=1}^{q} z_{l} \nabla h_{l}(u)\right)<0,
$$

which contradicts (2.1). Also, adding (2.8) and (2.10) and then applying hypothesis (b), we get (2.11). This contradicts to (2.1). Hence (2.3) cannot hold.

It is easy to derive the following result from the corresponding one by Egudo [7].

Corollary 2.2. Assume that the conclusion of Theorem 2.1 holds between (VOPE) and (MVODE). If $\left(u^{0}, \tau^{0}, w^{0}, y^{0}, z^{0}\right)$ is feasible for (MVODE) such that $u^{0}$ is feasible for (VOPE) and $u^{0 T} w_{i}^{0}=s\left(u^{0} \mid\right.$ $\left.C_{i}\right), i=1, \ldots, p$, then $u^{0}$ is efficient for (VOPE) and $\left(u^{0}, \tau^{0}, w^{0}, y^{0}, z^{0}\right)$ is efficient for (MVODE).

Theorem 2.3 (Strong Duality). If $x^{0}$ be efficient for (VOPE) and assume that $x^{0}$ satisfies a constraint qualification [14, pages 102-103] for (1.8) for at least one $k=1, \ldots, p$. Then there exist $\tau^{0} \in \mathbb{R}^{p}, y^{0} \in \mathbb{R}^{m}, z^{0} \in \mathbb{R}^{q}$ and $w_{i}^{0} \in C_{i}, i=1, \ldots, p$ such that $\left(x^{0}, \tau^{0}, w^{0}, y^{0}, z^{0}\right)$ is feasible for (MVODE) and $\left(x^{0}\right)^{T} w_{i}^{0}=s\left(x^{0} \mid C_{i}\right), i=1, \ldots, p$. If also weak duality (Theorem 2.1) holds between (VOPE) and (MVODE), then $\left(x^{0}, \tau^{0}, w^{0}, y^{0}, z^{0}\right)$ is efficient for (MVODE). 
Proof. Since $x^{0}$ is an efficient solution of (VOPE), by Lemma 1.4, $x^{0}$ solves (1.8) for each $k=$ $1, \ldots, p$. By hypothesis there exists at least one $k=1, \ldots, p$ such that $x^{0}$ satisfies a constraint qualification [14, pages 102,103] for (1.8). From the Kuhn-Tucker necessary conditions [14], we obtain $\tau_{i} \geqq 0$ for all $i \neq k, 0 \leqq y \in \mathbb{R}^{m}, z \in \mathbb{R}^{q}$ and $w_{i}^{0} \in C_{i}, i=1, \ldots, p$ such that

$$
\begin{gathered}
\nabla f_{k}\left(x^{0}\right)+w_{k}+\sum_{i \neq k} \tau_{i}\left(\nabla f_{i}\left(x^{0}\right)+w_{i}\right)+\sum_{j=1}^{m} y_{j} \nabla g_{j}\left(x^{0}\right)+\sum_{l=1}^{q} z_{l} \nabla h_{l}\left(x^{0}\right)=0, \\
w_{i}^{T} x^{0}=s\left(x^{0} \mid C_{i}\right), \quad i=1, \ldots, p, \\
\sum_{j=1}^{m} y_{j} g_{j}\left(x^{0}\right)=0 .
\end{gathered}
$$

Now dividing (2.12) and (2.14) by $1+\sum_{i \neq k} \tau_{i}$ and defining

$$
\begin{gathered}
\tau_{k}^{0}=\frac{1}{1+\sum_{i \neq k} \tau_{i}}>0, \quad \tau_{i}^{0}=\frac{\tau_{i}}{1+\sum_{i \neq k} \tau_{i}} \geqq 0, \\
y^{0}=\frac{y}{1+\sum_{i \neq k} \tau_{i}} \geqq 0, \quad z^{0}=\frac{z}{1+\sum_{i \neq k} \tau_{i}},
\end{gathered}
$$

we conclude that $\left(x^{0}, \tau^{0}, w^{0}, y^{0}, z^{0}\right)$ is feasible for (MVODE). The efficiency of $\left(x^{0}, \tau^{0}, w^{0}, y^{0}, z^{0}\right)$ for (MVODE) now follows from Corollary 2.2.

\section{Wolfe Type Duality}

We introduce a Wolfe type dual programming problem and establish weak and strong duality theorems.

$$
\begin{aligned}
\text { Maximize } & f(u)+u^{T} w+\left(y^{T} g(u)\right) e+\left(z^{T} h(u)\right) e \\
& =\left(f_{1}(u)+u^{T} w_{1}+y^{T} g(u)+z^{T} h(u), \ldots, f_{p}(u)+u^{T} w_{p}+y^{T} g(u)+z^{T} h(u)\right)
\end{aligned}
$$

(WVODE)

subject to

$$
\begin{aligned}
& \sum_{i=1}^{p} \tau_{i}\left(\nabla f_{i}(u)+w_{i}\right)+\nabla y^{T} g(u)+\nabla z^{T} h(u)=0, \\
& y_{j} \geqq 0, \quad j=1, \ldots, m, \quad w_{i} \in C_{i}, i=1, \ldots, p, \\
& \tau_{i} \geqq 0, \quad i=1, \ldots, p, \quad \sum_{i=1}^{p} \tau_{i}=1 .
\end{aligned}
$$


Theorem 3.1 (Weak Duality). Assume that for all feasible $x$ for (VOPE) and all feasible $(u, \tau, w, y, z)$ for (WVODE), $f_{i}(\cdot)+(\cdot)^{T} w_{i}, i=1, \ldots, p$, are $\rho_{i}$-convex, $g_{j}(\cdot), j=1, \ldots, m$, are $\sigma_{j}$-convex and $h_{l}(\cdot), l=1, \ldots, q$, are affine. If also any of the following conditions holds:

(a) $\tau_{i}>0$, forall $i \in P$ and $\sum_{i=1}^{p} \tau_{i} \rho_{i}+\sum_{j=1}^{m} y_{j} \sigma_{j} \geqq 0$;

(a) $\sum_{i=1}^{p} \tau_{i} \rho_{i}+\sum_{j=1}^{m} y_{j} \sigma_{j}>0$,

then the following cannot hold:

$$
\begin{gathered}
f_{i}(x)+s\left(x \mid C_{i}\right)<f_{i}(u)+u^{T} w_{i}+y^{T} g(u)+z^{T} h(u), \quad \text { for some } i \in P, \\
f_{i_{0}}(x)+s\left(x \mid C_{i_{0}}\right) \leqq f_{i_{0}}(u)+u^{T} w_{i_{0}}+y^{T} g(u)+z^{T} h(u), \quad \forall i_{0} \in P .
\end{gathered}
$$

Proof. Suppose contrary to the result that (3.3) hold. Then since $x$ is feasible for (VOPE) and $0 \leqq y \in \mathbb{R}^{m}, z \in \mathbb{R}^{q}$, (3.3) imply

$$
\begin{gathered}
f_{i}(x)+s\left(x \mid C_{i}\right)+y^{T} g(x)+z^{T} h(x)<f_{i}(u)+u^{T} w_{i}+y^{T} g(u)+z^{T} h(u), \quad \text { for some } i \in P, \\
f_{i_{0}}(x)+s\left(x \mid C_{i_{0}}\right)+y^{T} g(x)+z^{T} h(x) \leqq f_{i_{0}}(u)+u^{T} w_{i_{0}}+y^{T} g(u)+z^{T} h(u), \quad \forall i_{0} \in P .
\end{gathered}
$$

Since $x^{T} w_{i} \leqq s\left(x \mid C_{i}\right), i=1, \ldots, p,(3.4)$ yield

$$
\begin{gathered}
f_{i}(x)+x^{T} w_{i}+y^{T} g(x)+z^{T} h(x)<f_{i}(u)+u^{T} w_{i}+y^{T} g(u)+z^{T} h(u), \quad \text { for some } i \in P \\
f_{i_{0}}(x)+x^{T} w_{i_{0}}+y^{T} g(x)+z^{T} h(x) \leqq f_{i_{0}}(u)+u^{T} w_{i_{0}}+y^{T} g(u)+z^{T} h(u), \quad \forall i_{0} \in P .
\end{gathered}
$$

Now if hypothesis (a) holds, then from $\tau_{i}>0$ for all $i \in P$, (3.5) we obtain

$$
\begin{aligned}
& \sum_{i=1}^{p} \tau_{i}\left(f_{i}(x)+x^{T} w_{i}\right)+y^{T} g(x) \sum_{i=1}^{p} \tau_{i}+z^{T} h(x) \sum_{i=1}^{p} \tau_{i} \\
& \quad<\sum_{i=1}^{p} \tau_{i}\left(f_{i}(u)+u^{T} w_{i}\right)+y^{T} g(u) \sum_{i=1}^{p} \tau_{i}+z^{T} h(u) \sum_{i=1}^{p} \tau_{i}
\end{aligned}
$$

and since $\sum_{i=1}^{p} \tau_{i}=1$, this inequality reduces to

$$
\sum_{i=1}^{p} \tau_{i}\left(f_{i}(x)+x^{T} w_{i}-f_{i}(u)-u^{T} w_{i}\right)+y^{T} g(x)+z^{T} h(x)-y^{T} g(u)-z^{T} h(u)<0 .
$$


Now from (3.7), $\rho_{i}$-convexity of $f_{i}(\cdot)+(\cdot)^{T} w_{i}, i=1, \ldots, p, \sigma_{j}$-convexity of $g_{j}(\cdot), j=1, \ldots, m$ and $h_{l}(\cdot), l=1, \ldots, q$, is affine, we obtain

$$
(x-u)^{T}\left(\sum_{i=1}^{p} \tau_{i}\left(\nabla f_{i}(u)+w_{i}\right)+\nabla y^{T} g(u)+\nabla z^{T} h(u)\right)+\left(\sum_{i=1}^{p} \tau_{i} \rho_{i}+\sum_{j=1}^{m} y_{j} \sigma_{j}\right)\|x-u\|^{2}<0
$$

and since by hypothesis (a), $\sum_{i=1}^{p} \tau_{i} \rho_{i}+\sum_{j=1}^{m} y_{j} \sigma_{j} \geqq 0$, this implies

$$
(x-u)^{T}\left(\sum_{i=1}^{p} \tau_{i}\left(\nabla f_{i}(u)+w_{i}\right)+\nabla y^{T} g(u)+\nabla z^{T} h(u)\right)<0
$$

which contradicts (3.1). Also from (3.5), $\tau_{i} \geqq 0, i=1, \ldots, p$, and $\sum_{i=1}^{p} \tau_{i}=1$, we obtain

$$
\sum_{i=1}^{p} \tau_{i}\left(f_{i}(x)+x^{T} w_{i}-f_{i}(u)-u^{T} w_{i}\right)+y^{T} g(x)+z^{T} h(x)-y^{T} g(u)-z^{T} h(u) \leqq 0,
$$

and since $f_{i}(\cdot)+(\cdot)^{T} w_{i}, i=1, \cdots, p$, are $\rho_{i}$-convex, $g_{j}(\cdot), j=1, \ldots, m$, are $\sigma_{j}$-convex and $h_{l}(\cdot), l=1, \ldots, q$, are affine, (3.10) implies

$$
(x-u)^{T}\left(\sum_{i=1}^{p} \tau_{i}\left(\nabla f_{i}(u)+w_{i}\right)+\nabla y^{T} g(u)+\nabla z^{T} h(u)\right)+\left(\sum_{i=1}^{p} \tau_{i} \rho_{i}+\sum_{j=1}^{m} y_{j} \sigma_{j}\right)\|x-u\|^{2} \leqq 0 .
$$

Now by hypothesis (b), $\sum_{i=1}^{p} \tau_{i} \rho_{i}+\sum_{j=1}^{m} y_{j} \sigma_{j}>0$, hence (3.11) implies (3.9), again contradicting (3.1).

The following result can be easily driven from the corresponding one by Egudo [7].

Corollary 3.2. Assume that the conclusion of Theorem 3.1 holds between (VOPE) and (WVODE). If $\left(u^{0}, \tau^{0}, w^{0}, y^{0}, z^{0}\right)$ is feasible for (WVODE) such that $u^{0}$ is feasible for (VOPE), $y^{0 T} g\left(u^{0}\right)=0$ and $\left(u^{0}\right)^{T} w_{i}^{0}=s\left(u^{0} \mid C_{i}\right), i=1, \ldots, p$, then $u^{0}$ is efficient for (VOPE) and $\left(u^{0}, \tau^{0}, w^{0}, y^{0}, z^{0}\right)$ is efficient for (WVODE).

Theorem 3.3 (Strong Duality). If $x^{0}$ be efficient for (VOPE) and assume that $x^{0}$ satisfies a constraint qualification [14, pages 102,103] for (1.8) for at least one $k=1, \ldots, p$. Then there exist $\tau^{0} \in \mathbb{R}^{p}, y^{0} \in \mathbb{R}^{m}, z^{0} \in \mathbb{R}^{q}$ and $w_{i}^{0} \in C_{i}, i=1, \ldots, p$ such that $\left(x^{0}, \tau^{0}, w^{0}, y^{0}, z^{0}\right)$ is feasible for (WVODE) and $\left(y^{0}\right)^{T} g\left(x^{0}\right)=0,\left(x^{0}\right)^{T} w_{i}^{0}=s\left(x^{0} \mid C_{i}\right), i=1, \ldots, p$. If also weak duality (Theorem 3.1) holds between (VOPE) and (WVODE), then $\left(x^{0}, \tau^{0}, w^{0}, y^{0}, z^{0}\right)$ is efficient for 
(WVODE).

Proof. Since $x^{0}$ is an efficient solution of (VOPE), from Lemma 1.4, $x^{0}$ solves (1.8) for all $k=$ $1, \ldots, p$. By hypothesis there exists a $k \in P=\{1, \ldots, p\}$ for which $x^{0}$ satisfies a constraint qualification [14, pages 102-103] for (1.8). Now from the Kuhn-Tucker necessary conditions [14], there exist $\tau_{i} \geqq 0$ for all $i \neq k, 0 \leqq y \in \mathbb{R}^{m}, z \in \mathbb{R}^{q}$ and $w_{i}^{0} \in C_{i}, i=1, \ldots, p$ such that

$$
\begin{gathered}
\nabla f_{k}\left(x^{0}\right)+w_{k}+\sum_{i \neq k} \tau_{i}\left(\nabla f_{i}\left(x^{0}\right)+w_{i}\right)+\sum_{j=1}^{m} y_{j} \nabla g_{j}\left(x^{0}\right)+\sum_{l=1}^{q} z_{l} \nabla h_{l}\left(x^{0}\right)=0, \\
w_{i}^{T} x^{0}=s\left(x^{0} \mid C_{i}\right), \quad i=1, \ldots, p, \\
\sum_{j=1}^{m} y_{j} g_{j}\left(x^{0}\right)=0
\end{gathered}
$$

Now dividing (3.12) and (3.14) by $1+\sum_{i \neq k} \tau_{i}$ and defining

$$
\begin{gathered}
\tau_{k}^{0}=\frac{1}{1+\sum_{i \neq k} \tau_{i}}>0, \quad \tau_{i}^{0}=\frac{\tau_{i}}{1+\sum_{i \neq k} \tau_{i}} \geqq 0, \\
y^{0}=\frac{y}{1+\sum_{i \neq k} \tau_{i}} \geqq 0, \quad z^{0}=\frac{z}{1+\sum_{i \neq k} \tau_{i}},
\end{gathered}
$$

we conclude that $\left(x^{0}, \tau^{0}, w^{0}, y^{0}, z^{0}\right)$ is feasible for (WVODE).

The efficiency of $\left(x^{0}, \tau^{0}, w^{0}, y^{0}, z^{0}\right)$ for (WVODE) now follows from Corollary 3.2.

\section{Special Cases}

We give some special cases of our duality results.

(1) If support functions are excepted and $h=0$, then our dual programs are reduced to the duals in Egudo [7].

(2) Let $C_{i}=\left\{B_{i} w: w^{T} B_{i} w \leqq 1\right\}$. Then $s\left(x \mid C_{i}\right)=\left(x^{T} B_{i} x\right)^{1 / 2}$ and the sets $C_{i}, i=$ $1, \ldots, p$, are compact and convex. If $h=0$, then (VOPE), (MVODE) and (WVODE) reduce to the corresponding $(\mathrm{VP}),(\mathrm{VDP})_{2}$ and $(\mathrm{VDP})_{1}$ in Lal et al. [8], respectively.

(3) If we replace $\rho$-convexity by generalized $(F, \rho)$-convexity, then our weak duality theorems reduce to the corresponding ones in Yang et al. [11].

\section{References}

[1] A. M. Geoffrion, "Proper efficiency and the theory of vector maximization," Journal of Mathematical Analysis and Applications, vol. 22, pp. 618-630, 1968.

[2] T. Weir, "Proper efficiency and duality for vector valued optimization problem," Journal of the Australian Mathematical Society, Series A, vol. 43, pp. 21-34, 1987.

[3] P. Wolfe, "A duality theorem for nonlinear programming," Quarterly of Applied Mathematics, vol. 19, pp. 239-244, 1961. 
[4] J. P. Vial,, "Strong convexity of sets and functions," Journal of Mathematical Economics, vol. 9, pp. 187205, 1982.

[5] J. P. Vial, "Strong and weak convexity of sets and functions," Mathematics of Operations Research, vol. 8, pp. 231-259, 1983.

[6] R. R. Egudo, "Proper efficiency and multiobjective duality in nonlinear programming," Journal of Information and Optimization Sciences, vol. 8, pp. 155-166, 1987.

[7] R. R. Egudo, "Efficiency and generalized convex duality for multiobjective programs," Journal of Mathematical Analysis and Applications, vol. 138, no. 1, pp. 84-94, 1989.

[8] S. N. Lal, B. Nath, and A. Kumar, "Duality for some nondifferentiable static multiobjective programming problems," Journal of Mathematical Analysis and Applications, vol. 186, pp. 862-867, 1994.

[9] B. Mond and M. Schechter, "Non-differentiable symmetric duality," Bulletin of the Australian Mathematical Society, vol. 53, pp. 177-187, 1996.

[10] H. Kuk, G. M. Lee, and D. S. Kim, "Nonsmooth multiobjective programs with V- $\rho$-invexity," Indian Journal of Pure E Applied Mathematics, vol. 29, pp. 405-415, 1998.

[11] X. M. Yang, K. L. Teo, and X. Q. Yang, "Duality for a class of nondifferentiable multiobjective programming problem," Journal of Mathematical Analysis and Applications, vol. 252, pp. 999-1005, 2000.

[12] D. S. Kim and K. D. Bae, "Optimality conditions and duality for a class of nondifferentiable multiobjective programming problems," Taiwanese Journal of Mathematics, vol. 13, no. 2B, pp. 789-804, 2009.

[13] V. Chankong and Y. Y. Haimes, Multiobjective Decision Making: Theory and Methodology, vol. 8 of NorthHolland Series in System Science and Engineering, North-Holland, New York, NY, USA, 1983.

[14] O. L. Mangasarian, Nonlinear Programming, McGraw-Hill, New York, NY, USA, 1969. 